\section{(6) OPEN ACCESS}

\title{
The gut microbiota plays a protective role in the host defence against pneumococcal pneumonia
}

\author{
Tim J Schuijt, ${ }^{1,2,3}$ Jacqueline M Lankelma, ${ }^{1}$ Brendon P Scicluna, ${ }^{1}$ \\ Felipe de Sousa e Melo, ${ }^{1}$ Joris J T H Roelofs, ${ }^{4}$ J Daan de Boer, ${ }^{1}$ Arjan J Hoogendijk, \\ Regina de Beer, ${ }^{1}$ Alex de Vos, ${ }^{1}$ Clara Belzer, ${ }^{5}$ Willem M de Vos, ${ }^{5,6}$ \\ Tom van der Poll, ${ }^{1,2}$ W Joost Wiersinga ${ }^{1,2}$
}

\begin{abstract}
- Additional material is published online only. To view please visit the journal online (http://dx.doi.org/10.1136/ gutjnl-2015-309728).

For numbered affiliations see end of article.

\section{Correspondence to} Dr W Joost Wiersinga, Department of Medicine Division of Infectious Diseases, Center for Experimental and Molecular Medicine, Academic Medical Center, University of Amsterdam, Meibergdreef 9, Room G2-130, Amsterdam 1105 AZ, The Netherlands; w.j.wiersinga@amc.uva.nl.
\end{abstract}

Received 3 April 2015 Revised 18 August 2015 Accepted 20 August 2015 Published Online First 28 October 2015

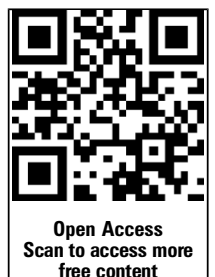

\section{SLinked}

- http://dx.doi.org/10.1136/ gutjnl-2015-310599

\section{CrossMark}

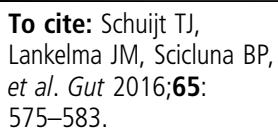

To cite: Schuijt TJ, Lankelma JM, Scicluna BP et al. Gut 2016;65: 575-583.

\section{ABSTRACT}

Objective Pneumonia accounts for more deaths than any other infectious disease worldwide. The intestinal microbiota supports local mucosal immunity and is increasingly recognised as an important modulator of the systemic immune system. The precise role of the gut microbiota in bacterial pneumonia, however, is unknown. Here, we investigate the function of the gut microbiota in the host defence against Streptococcus pneumoniae infections.

Design We depleted the gut microbiota in C57BL/6 mice and subsequently infected them intranasally with $S$. pneumoniae. We then performed survival and faecal microbiota transplantation (FMT) experiments and measured parameters of inflammation and alveolar macrophage whole-genome responses.

Results We found that the gut microbiota protects the host during pneumococcal pneumonia, as reflected by increased bacterial dissemination, inflammation, organ damage and mortality in microbiota-depleted mice compared with controls. FMT in gut microbiota-depleted mice led to a normalisation of pulmonary bacterial counts and tumour necrosis factor- $\alpha$ and interleukin-10 levels $6 \mathrm{~h}$ after pneumococcal infection. Whole-genome mapping of alveolar macrophages showed upregulation of metabolic pathways in the absence of a healthy gut microbiota. This upregulation correlated with an altered cellular responsiveness, reflected by a reduced responsiveness to lipopolysaccharide and lipoteichoic acid. Compared with controls, alveolar macrophages derived from gut microbiota-depleted mice showed a diminished capacity to phagocytose S. pneumoniae. Conclusions This study identifies the intestinal microbiota as a protective mediator during pneumococcal pneumonia. The gut microbiota enhances primary alveolar macrophage function. Novel therapeutic strategies could exploit the gut-lung axis in bacterial infections.

\section{INTRODUCTION}

Pneumonia accounts for more deaths than any other infectious disease worldwide. ${ }^{1} 2$ It is estimated that every year nearly 12 million children alone are hospitalised for severe pneumonia. ${ }^{2}$ Streptococcus pneumoniae, a Gram-positive bacteria that colonises the upper respiratory tract, is the most frequent cause of community-acquired pneumonia and a significant cause of morbidity

\section{Significance of this study}

What is already known on this subject?

- The gut microbiota can enhance local defences against enteral pathogens and is also believed to influence systemic immunity.

- Gut microbiota metabolism of dietary fibres influences the severity of allergic inflammation in mice.

- The role of the gut microbiota in the host response to bacterial pneumonia is ill-defined.

What are the new findings?

- The present study describes the role of the intestinal microbiota in the host defence against bacterial pneumonia.

- Mice with a depleted gut microbiota have increased bacterial dissemination, inflammation, organ failure and an accelerated mortality when compared with controls following infection with Streptococcus pneumoniae, indicating that the intestinal microbiota acts as a protective factor in the host defence against pneumococcal pneumonia.

- Faecal microbiota transplantation to gut microbiota-depleted mice led to a normalisation of pulmonary bacterial counts and tumour necrosis factor- $\alpha$ and interleukin-10 levels $6 \mathrm{~h}$ after pneumococcal infection.

- The gut microbiota has a marked influence on metabolic pathways within alveolar macrophages, which correlates with an altered cellular responsiveness. Macrophages in microbiota-depleted mice have a diminished capacity to phagocytose S. pneumoniae and demonstrate a reduced cellular responsiveness towards lipoteichoic acid and lipopolysaccharide.

and mortality worldwide. ${ }^{3}{ }^{4}$ Growing levels of bacterial resistance have complicated the treatment of pneumococcal infections. Thus, there is an urgent need to expand our knowledge of the pathogenesis of severe pneumonia caused by this common pathogen. 
Significance of this study

\section{How might it impact on clinical practice in the} foreseeable future?

- Our data identify a gut-lung axis during infection and establish a mechanism for pulmonary immunomodulation via the intestinal microbiota. These results highlight the possibility that broad-spectrum antibiotics and disruption of the intestinal microbiota may diminish innate immune defences to infection. Novel therapies to treat severe pneumonia could focus on the gut-lung axis in bacterial infection.

For many years, it was hypothesised that in critically ill patients the intestine plays a detrimental role by promoting systemic inflammation and infection. ${ }^{5}$ More recently, however, the intestinal microbiota-consisting of more bacteria than the total number of cells in the human body-has emerged as positive player in the host defence system, supporting mucosal immunity and potentially modulating systemic immunity. ${ }^{6-13}$ The intestinal microbiota may be of profound clinical relevance, particularly for intensive care medicine where the majority of patients are treated with antibiotics, which have pervasive and long-term effects on their intestinal microbiota. ${ }^{9}{ }^{14}$ What role does gut microbiota play in bacterial pneumonia, however, remains elusive.

We hypothesised that the gut microbiota bolsters the host defence against $S$. pneumoniae. Therefore, we depleted the gut microbiota in mice and studied subsequent infection with $S$. pneumoniae. We provide evidence that the intestinal microbiota acts as a protective factor in the host defence against pneumococcal pneumonia and show that the gut microbiota enhances primary alveolar macrophage function. Our studies provide a basis for novel therapeutic strategies that exploit the gut-lung axis in bacterial infection.

\section{RESULTS}

\section{Protective role of the gut microbiota during pneumococcal pneumonia}

To first gain insight into the role the intestinal microbiota plays during bacterial pneumonia, we first treated wild-type mice with broad-spectrum antibiotics (ampicillin, neomycin, metronidazole and vancomycin) in their drinking water in order to deplete the gut microbiota. ${ }^{7}$ We then intranasally challenged them with S. pneumoniae $\left(10^{6}\right.$ colony forming units (CFU); figure $\left.1 \mathrm{~A}\right)$. Microbiota-depleted mice had an accelerated mortality rate following S. pneumoniae infection (figure 1B). Supporting this finding, gut microbiota-depleted mice had increased bacterial loads in their lungs $6 \mathrm{~h}$ after $S$. pneumoniae challenge when compared with age-matched controls and gut microbiota mice had more bacteria in their blood $48 \mathrm{~h}$ post-infection (figure 1C, D). Because the ability to combat invading pathogens strongly depends on the efficacy of the local inflammatory response, ${ }^{15}$ we measured the abundance of pulmonary cytokines and chemokines. Interleukin (IL)-1 $1 \beta$, IL-6 and CXCL1 were increased, whereas tumour necrosis factor (TNF)- $\alpha$ and IL-10 levels were decreased $6 \mathrm{~h}$ after intranasal S. pneumoniae infection in the gut microbiota-depleted mice compared with controls (table 1). Lung cytokine levels at later time points were similar between groups (table 1).
Faecal microbiota transplantation to gut microbiota-depleted mice restores pulmonary bacterial clearance and TNF- $\alpha$ and IL-10 levels early after pneumococcal infection

We next aimed to verify that our mortality, bacterial load and cytokine findings were gut microbiota dependent. Therefore, we studied the effects orally administering intestinal microbiota from healthy mice to microbiota-depleted mice had on the recipients' response to $S$. pneumoniae infection. We first established that broad-spectrum antibiotic treatment did not influence gut architecture or epithelial integrity (figure 1E). In addition, we tested whether the lung barrier might be impaired in the absence of a healthy microbiota by measuring bronchoalveolar lavage fluid (BALF) protein contents. No differences in BALF protein contents were observed after antibiotic treatment (see online supplementary figure S1). In correspondence with our hypothesis, faecal microbiota transplantation (FMT) to gut microbiota-depleted mice could restore the diminished bacterial clearance in the lung early after pneumococcal infection (figure 1F). In addition, FMT led to a normalisation of TNF- $\alpha$ and IL-10 levels in the lung $6 \mathrm{~h}$ after infection, comparable with non-depleted control mice (table 2). However, faecal transplantation did not significantly affect the number of $S$. pneumoniae present in the blood when compared with antibiotic-treated mice or controls (data not shown) nor did it significantly affect IL-1, IL-6 or CXCL1 levels (table 2). To assess the magnitude by which our antibiotics ${ }^{7}$ reduced bacterial numbers in the gut, we evaluated the murine faeces using a phylogenetic microarray analysis of over 16000 intestinal small subunit ribosomal RNA sequences and determined the diversity index. ${ }^{16}{ }^{17}$ Antibiotic treatment led to a significant drop in the microbial diversity, which was significantly reversed by transplantation of normal faeces (figure 1G-I). Unsupervised clustering and principal component analysis of the samples indicates a clear partitioning between controls, antibiotic-treated and faecal transplantation groups, which can explain $83.4 \%$ of the total data variance (figure 1G-I). Taken together, these data establish that gut microbiota depletion leads to a detrimental host response to pneumococcal pneumonia as reflected by increased bacterial counts and reduced survival.

\section{The gut microbiota protects against organ damage during S. pneumoniae-induced sepsis}

To determine whether the protective role of the gut microbiota on the pulmonary antibacterial host defence extended to organ damage, we semiquantitatively scored lung histology slides from gut microbiota-depleted mice and controls at various time points after S. pneumoniae challenge. All infected mice showed histological evidence of severe pneumonia. Consistent with the observed increased bacterial outgrowth, at $6 \mathrm{~h}$ post-infection microbiota-depleted mice displayed earlier and significantly more inflammation in their lungs compared with controls, demonstrated by enhanced interstitial inflammation, endothelialitis and oedema (figure 2A). This difference in pulmonary organ damage was even more pronounced at 24 and $48 \mathrm{~h}$ (figure 2B, C). Since recruitment of leucocytes to infectious sites is an essential step in the host's defence against pneumonia, ${ }^{3}$ we next analysed Gr-1 stainings in lung tissue and found increased neutrophil influx in the gut microbiota-depleted mice (see online supplementary figure S2). Depletion of the intestinal microbiota during $S$. pneumoniae infection also resulted in an enhanced detrimental systemic inflammatory response. Although no differences in systemic cytokine levels (TNF- $\alpha$, IL-6, IL-10, 
A

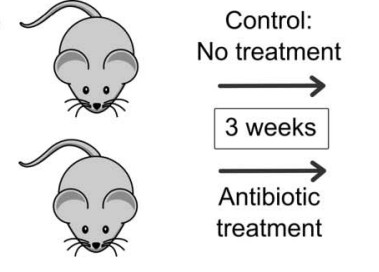

B

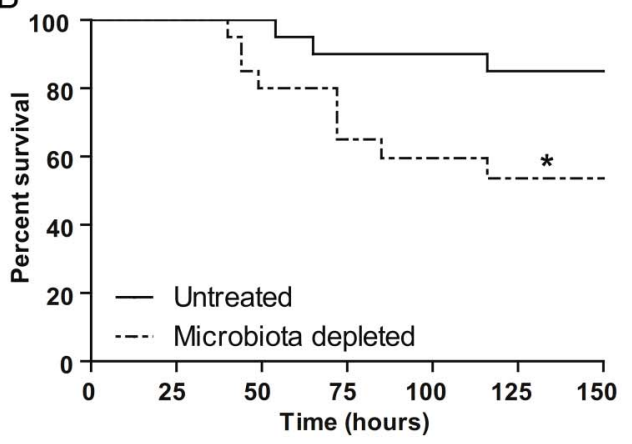

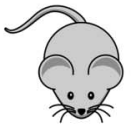

Stop treatment for 2 days
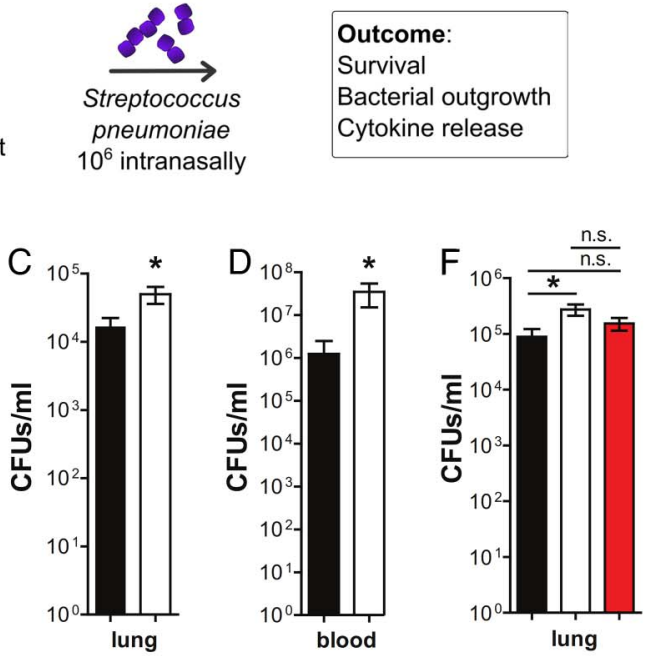

Untreated $\square$ Microbiota depleted
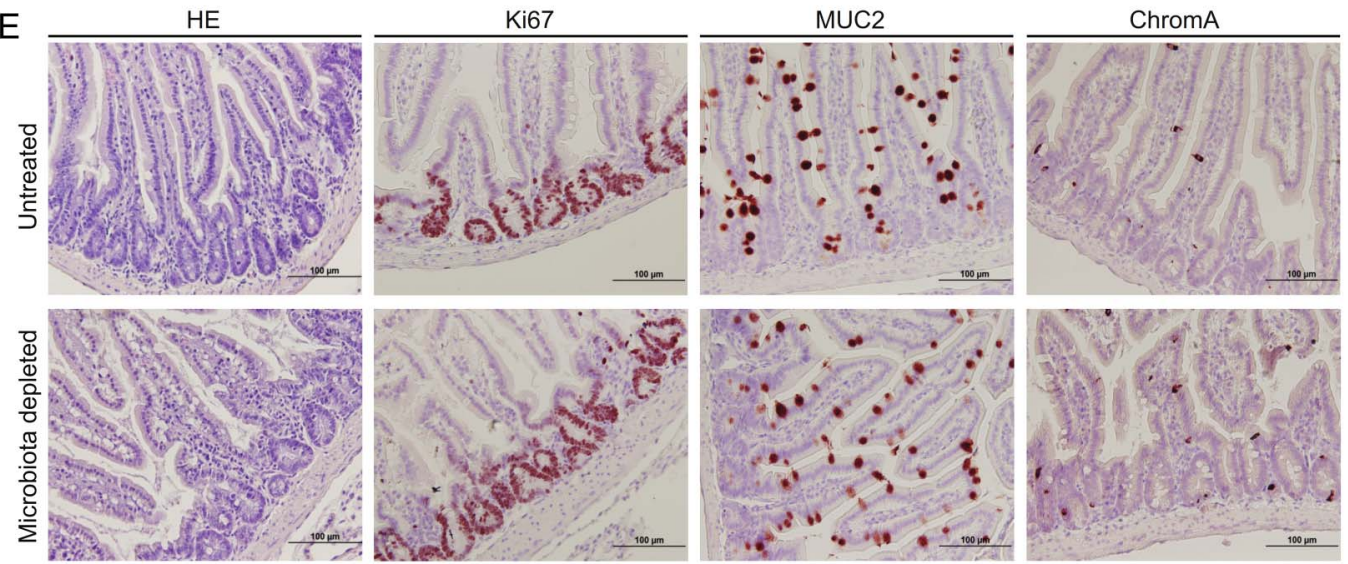

G

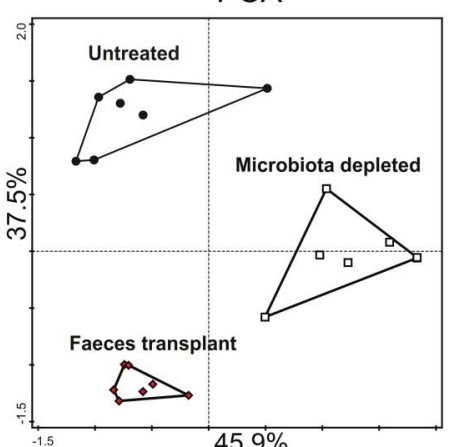

H Cluster Dendrogram

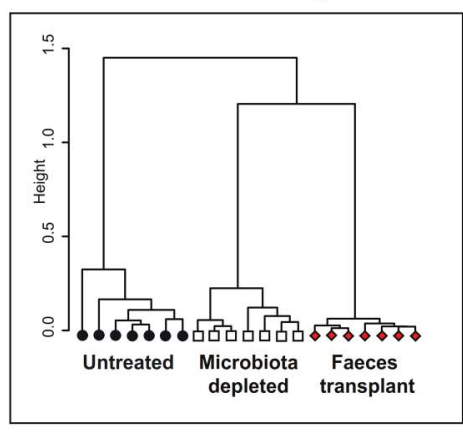

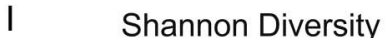

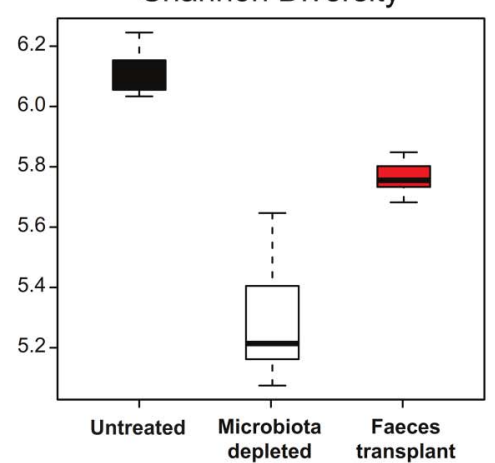

Figure 1 Protective role of the gut microbiota during pneumococcal pneumonia. (A) Experimental design. Group of eight wild-type mice were treated for 3 weeks with broad-spectrum antibiotics (ampicillin, neomycin, metronidazole and vancomycin) in their drinking water compared with untreated controls. Two days post treatment mice received an intranasal challenge with $1 \times 10^{6}$ colony forming units (CFU) of Streptococcus pneumoniae. Subsequently, mouse survival, bacterial outgrowth and cytokine release were determined at various time points post $S$. pneumoniae infection. (B) Survival of mice treated with broad-spectrum antibiotics compared with untreated controls before intranasal challenge with $1 \times 10^{6} \mathrm{CFU}$ of S. pneumoniae. (C) Pulmonary bacterial counts $6 \mathrm{~h}$ after S. pneumoniae infection in untreated (black) and microbiota-depleted (white) mice. (D) Blood bacterial counts $48 \mathrm{~h}$ after S. pneumoniae infection in untreated (black) and microbiota-depleted (white) mice. (E) Representative small intestine sections of untreated (upper row) and microbiota-depleted (lower row) mice demonstrate intact epithelial integrity with H\&E, Ki67 (proliferation restricted in the crypt), MUC2 (goblet cell differentiation) and ChromograninA (neuroendocrine cell differentiation) stainings in both groups. (F) Effect of faecal microbiota transplantation to gut microbiota-depleted mice on lung bacterial counts $6 \mathrm{~h}$ after intranasal $S$. pneumoniae infection. (G) The magnitude by which the antibiotic protocol depleted the gut microbiota was assessed using a phylogenetic microarray in which microbiota composition samples were clustered based on principal component analysis (PCA), (H) Pearson Clustering and (I) the Shannon Diversity Index. Group size is 8-12 per group; results are shown as means \pm SEM; n.s. denotes not significant; * $\mathrm{p}<0.05$. 
Table 1 Pulmonary cytokine concentrations in untreated and microbiota-depleted mice during pneumococcal pneumonia

\begin{tabular}{|c|c|c|c|}
\hline & \multicolumn{3}{|c|}{ Time after Streptococcus pneumoniae infection } \\
\hline & $6 \mathrm{~h}$ & $24 \mathrm{~h}$ & $48 \mathrm{~h}$ \\
\hline \multicolumn{4}{|l|}{ TNF- $\alpha$} \\
\hline Untreated & $2038(1306-2130)$ & $1950(1722-2449)$ & $123.6(109.5-174.9)$ \\
\hline Microbiota depleted & $1021(707.3-1091)^{* *}$ & $1074(929.4-1277)^{* *}$ & $152.3(134.5-494.5)$ \\
\hline \multicolumn{4}{|l|}{ IL-1 } \\
\hline Untreated & $96.6(52.6-106.5)$ & $62.4(57.9-236.1)$ & $48.7(43.5-53.7)$ \\
\hline Microbiota depleted & $120.9(89.7-128.2)^{*}$ & $109.3(80.0-204.8)$ & $64.5(47.7-122.0)$ \\
\hline \multicolumn{4}{|l|}{ IL-6 } \\
\hline Untreated & $71.4(42.0-86.5)$ & $80.4(42.1-538.4)$ & $105.2(98.4-125.5)$ \\
\hline Microbiota depleted & $110.8(90.2-152.9)^{*}$ & $100.7(52.2-192.9)$ & $117.1(99.6-312.8)$ \\
\hline \multicolumn{4}{|l|}{ IL-10 } \\
\hline Untreated & $41.3(29.9-60.84)$ & $75.7(45.0-91.4)$ & $48.4(30.7-77.3)$ \\
\hline Microbiota depleted & $21.4(10.5-27.4)^{*}$ & $45.2(19.6-52.9)$ & $51.43(43.7-80.4)$ \\
\hline \multicolumn{4}{|l|}{ CXCL1 } \\
\hline Untreated & $125.5(68.5-147.4)$ & $87.8(60.4-839.1)$ & $54.3(44.5-226.8)$ \\
\hline Microbiota depleted & $247.3(155.1-455.1)^{* *}$ & $132.1(59.1-448.1)$ & $44.3(23.9-134.8)$ \\
\hline
\end{tabular}

IL-12, IFN- $\gamma$ and MCP-1) could be observed between groups (see online supplementary figure S3), $48 \mathrm{~h}$ after infection gut microbiota-depleted mice had enhanced tissue inflammation and damage (liver figure 2D,E) and hepatic injury as reflected by elevated plasma concentration of aspartate aminotransferase, alanine aminotransferase and lactate dehydrogenase (figure 2F-I). Kidney function, as measured by urea and creatinine, was not affected (figure $2 \mathrm{~F}$ and see online supplementary figure S4). These results suggest that the gut microbiota protects against organ injury during $S$. pneumoniae-induced sepsis.

\section{Effect of intestinal microbiota depletion on the alveolar macrophage transcriptome}

Because it has been described that the intestinal microbiota can constitutively prime bone marrow-derived neutrophils and augment their capacity to kill microorganisms, ${ }^{7}$ we further hypothesised that a similar axis exists between the gut microbiota and alveolar macrophages, which are the major orchestrators of

Table 2 Pulmonary cytokine concentrations in untreated and microbiota-depleted mice with and without faecal microbiota transplantation during pneumococcal pneumonia

\begin{tabular}{lclc}
\hline & Untreated & Microbiota depleted & Faeces transplant \\
\hline TNF- $\alpha$ & $878.5(588.5-1185)$ & $552.9(476.8-764.2)^{*}$ & $858.9(660.4-1564)$ \\
IL-1 & $122(61.59-166.8)$ & $232.5(143.5-342.1)^{*}$ & $217(129-307.1)^{*}$ \\
IL-6 & $198.9(95.72-240.2)$ & $419.4(279.2-555.6)^{* *}$ & $326.8(210.8-454.7)^{*}$ \\
IL-10 & $227.7(160-300.3)$ & $123.7(116.8-128.1)^{*}$ & $174.4(153.7-189)$ \\
CXCL1 & $144.6(84.43-223.7)$ & $384.4(260.3-533.9)^{* * *}$ & $242.9(185.4-271)^{*}$
\end{tabular}

Data are expressed as median (IQR) of $n=8$ mice per group per time point.

Measurements are expressed in $\mathrm{pg} / \mathrm{mL}$.

${ }^{*} \mathrm{p}<0.05$ for Untreated versus Microbiota depleted or for Untreated versus Faeces transplant mice (Mann-Whitney U test).

${ }^{* *} p<0.01$ for Untreated versus Microbiota depleted or for Untreated versus Faeces transplant mice (Mann-Whitney U test).

${ }^{* * *} p<0.001$ for Untreated versus Microbiota depleted or for Untreated versus Faeces transplant mice (Mann-Whitney $\mathrm{U}$ test).

CXCL1, (C-X-C motif) ligand 1; IL, interleukin; TNF- $\alpha$, a tumour necrosis factor- $\alpha$. the pulmonary immune response following pathogen invasion. ${ }^{4} 15$ To test this hypothesis and to define the mechanism by which the gut microbiota exerts its protective effects during pneumonia, we first analysed genome-wide transcriptional responses in alveolar macrophages. Alveolar macrophages were isolated from uninfected but gut microbiota-depleted mice and controls. Depletion of the intestinal microbiota had a substantial effect on the alveolar macrophage transcriptome: considering multiple comparison-corrected (Benjamini-Hochberg) $\mathrm{p}$ values, 80 unique genes were found to discriminate between alveolar macrophages derived from gut microbiota-depleted mice and controls (figure $3 \mathrm{~A}$ ). Ingenuity pathway analysis revealed enrichment of the superpathway of cholesterol biosynthesis and zymosterol biosynthesis canonical signalling pathways (figure 3B). Genes within these pathways include Idi1, encoding isopentenyl-diphosphate-delta-isomerise-1 and Acat 2, encoding acetyl-Coenzyme-A-acetyltransferase-2. These transcriptome differences suggest marked alterations in the metabolic status of lung alveolar macrophages after gut microbiota depletion.

\section{The gut microbiota enhances primary alveolar macrophage function}

Cholesterol biosynthesis plays an important role in the antibacterial effector functions of alveolar macrophages. ${ }^{18} 19$ Blockade of cholesterol synthesis significantly inhibits the function of cholesterol-rich membrane rafts and as a result the phagocytosis of Pseudomonas aeruginosa by alveolar macrophages. ${ }^{19}$ Alveolar macrophage phagocytosis is one of the prime host defence mechanisms during pneumococcal infection and pneumonia. ${ }^{4} 15$ Given the observed effect of gut microbiota depletion on the cholesterol biosynthesis pathway, we next studied whether gut microbiota depletion altered the phagocytosis of $S$. pneumoniae by alveolar macrophages. To do this, we harvested primary alveolar macrophages from uninfected gut microbiota-depleted and control mice and compared their ability to internalise CFSE-labelled S. pneumoniae. We found that alveolar macrophages derived from gut microbiota-depleted mice had a diminished capacity to phagocytose $S$. pneumoniae when compared 
Figure 2 The gut microbiota protects against organ failure during Streptococcus pneumoniae-induced sepsis. (A-C) Representative lung slides of untreated (left) and microbiota-depleted (right) mice infected with $1 \times 10^{6}$ colony forming units (CFU) of $S$. pneumoniae via the intranasal route and euthanised at indicated time points $(6,24$ and $48 \mathrm{~h}$ ) thereafter to assess pulmonary inflammation and total lung histopathology scores (see Materials and methods). H\&E staining; original magnification, $\times 100$. ( $D$ and $E$ ) Liver and spleen histology shown $48 \mathrm{~h}$ after infection. H\&E staining; original magnification, $\times 200$ for liver, $\times 100$ for spleen. (F) Systemic blood urea nitrogen (BUN), (G) aspartate aminotransferase (AST), (H) alanine aminotransferase (ALT) and (I) lactate dehydrogenase (LDH) levels assessed $48 \mathrm{~h}$ after infection in untreated (black) and gut microbiota-depleted (white) mice. Group size is 8 per group; results are shown as means \pm SEM; n.s. denotes not significant; ${ }^{*} \mathrm{p}<0.05$ and ${ }^{* *} p<0.01$.
A

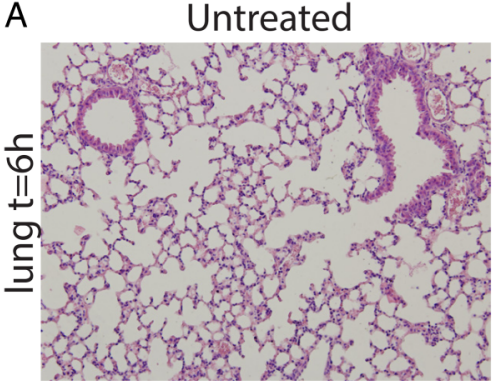

B
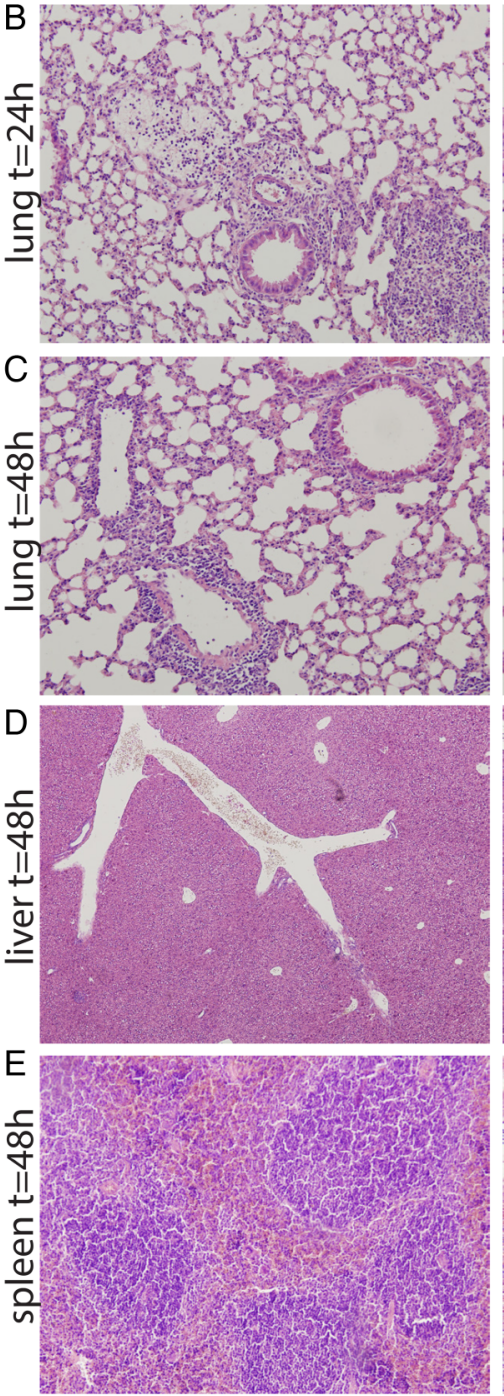

$\mathrm{F}$

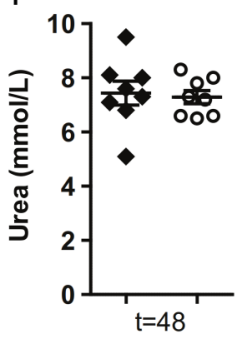

G

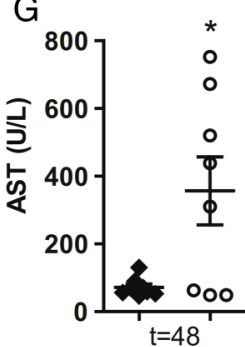

Microbiota depleted
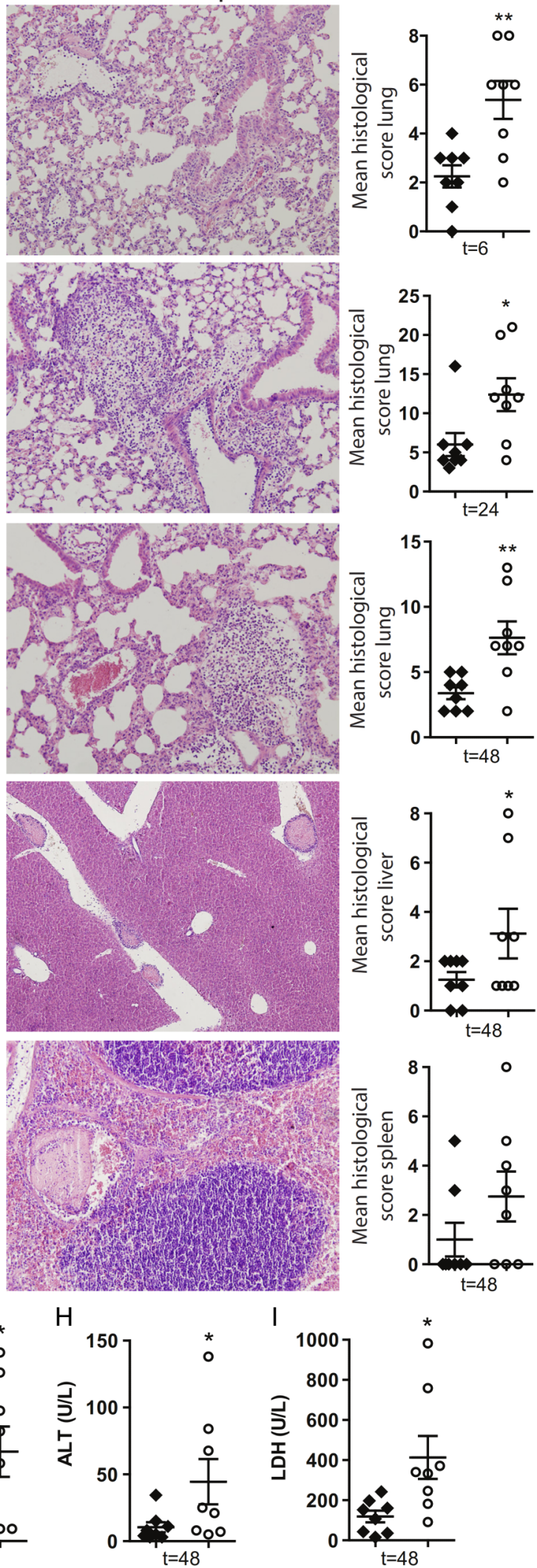

with controls, which may explain the in vivo phenotype observed in the gut microbiota-depleted mice (figure 4A). Gut microbiota-dependent phagocytosis was compartment dependent: whole-blood neutrophils (figure 4B), but not peritoneal macrophages (figure 4C) derived from microbiota-depleted mice showed an equal defect in their phagocytosis capacity compared with controls. Responsiveness of alveolar macrophages derived from gut microbiota-depleted mice towards lipoteichoic acid (LTA; figure 4D, E) or lipopolysaccharide (LPS; figure 4F, G) was also markedly diminished compared with controls in terms 

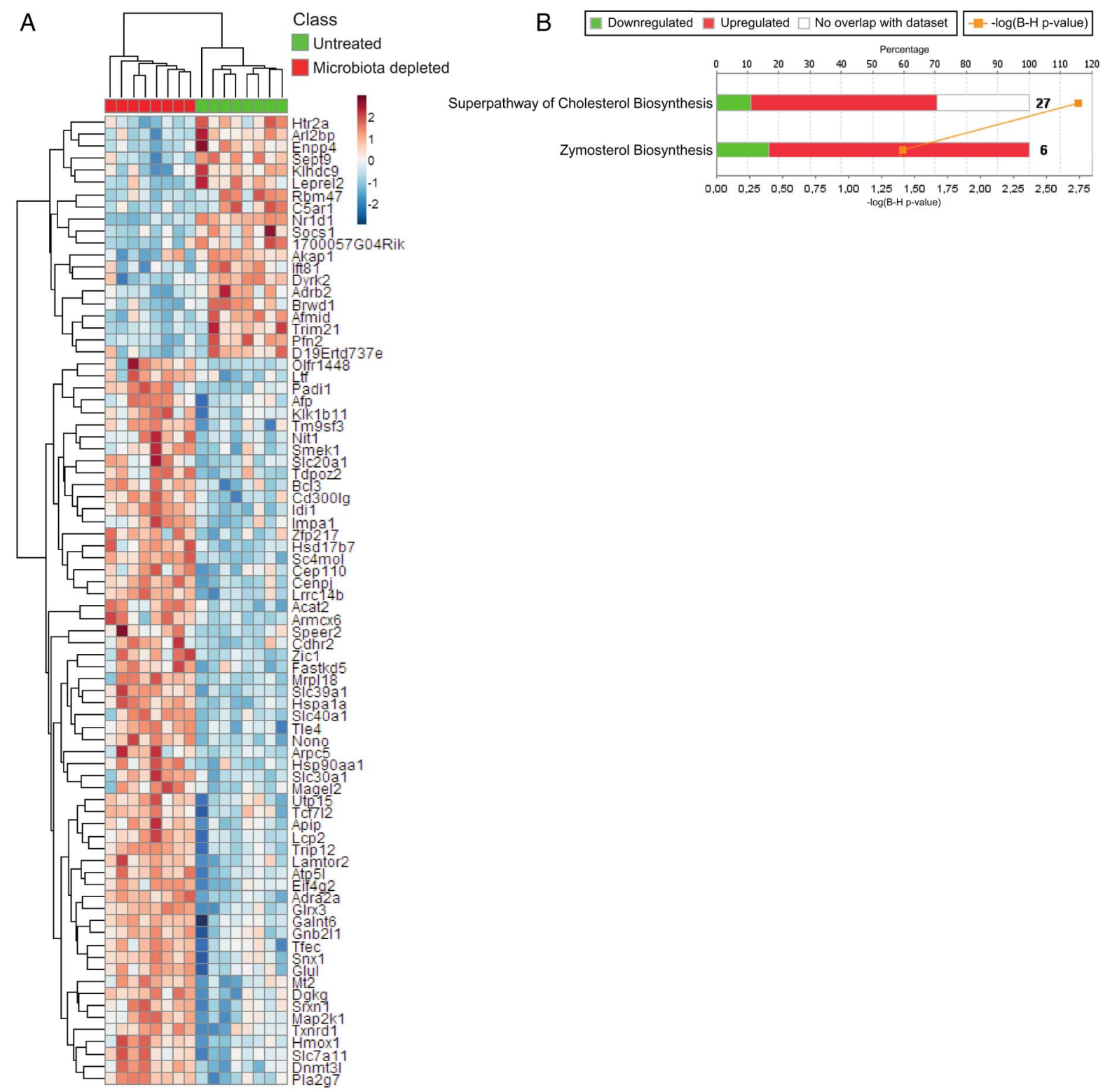

Figure 3 Effect of intestinal microbiota depletion on the alveolar macrophage transcriptome. (A) Unsupervised hierarchical clustering heatmap of the significant (multiple comparison adjusted $p<0.05$ ) differentially expressed genes between untreated control and gut microbiota-depleted lung alveolar macrophages. Red denotes increased expression; blue denotes decreased expression. (B) Stacked bar plot depicting the significantly enriched canonical signalling pathways (ingenuity pathway analysis) and expression patterns. $-\log (B-H) p$, negative log-transformed BenjaminiHochberg-adjusted Fisher test $p$ value. Ratio, ratio of input genes to pathway genes. Red denotes increased expression in gut microbiota-depleted alveolar macrophages; green denotes decreased expression in gut microbiota-depleted alveolar macrophages.

of cytokine release. The observed effect of the gut microbiota was compartment dependent; whole blood (figure $4 \mathrm{H}$ ), but not peritoneal macrophages derived from gut microbiota-depleted mice showed a diminished responsiveness towards LTA or LPS when compared with controls (figure 4I-L). Taken together, these data confirm that the gut microbiota enhances primary alveolar macrophage function and that an unperturbed gut microbiota enhances responsiveness to bacterial virulence factors and increases phagocytosis capacity.

\section{DISCUSSION}

Each year, 3.5 million deaths are attributed to pneumonia, although this number is probably an underestimation, since deaths from sepsis and deaths attributed to other conditions (eg, cancer and Alzheimer's disease) for which pneumonia is the terminal event are coded separately. ${ }^{1}{ }^{20}$ Pneumonia is the most common cause of sepsis and S. pneumoniae is the most frequently isolated pathogen in pneumonia resulting in sepsis. Recent breakthroughs in our understanding of the role of the gut microbiome in both health and disease can therefore have considerable implications for respiratory and critical care medicine. ${ }^{911-13}$ Here, we show that the gut microbiota plays a protective role during $S$. pneumoniae pneumonia as evidenced by the increased bacterial dissemination, inflammation, organ failure and mortality in microbiota-depleted mice compared with controls. FMT to gut microbiota-depleted mice led to a normalisation of pulmonary bacterial counts and TNF- $\alpha$ and IL-10 levels $6 \mathrm{~h}$ after pneumococcal infection. The gut microbiota enhances primary alveolar macrophage function both in terms of cellular responsiveness to LTA and LPS and the capacity to phagocytose S. pneumoniae.

Our work underscores the concept that the large community of intestinal microbes not only contributes to the local host 

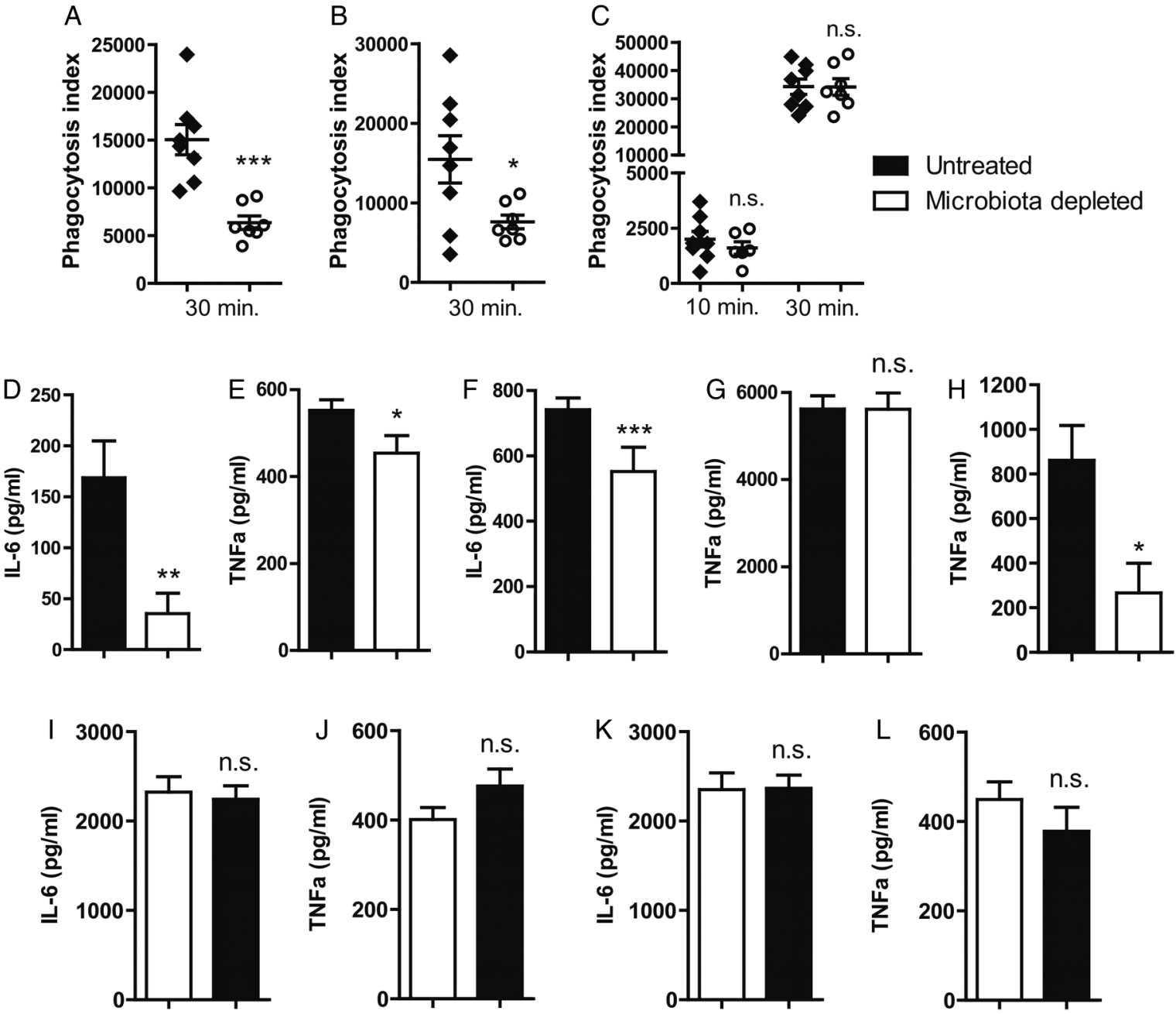

Figure 4 The gut microbiota enhances primary alveolar macrophage function. (A) Capacity of alveolar macrophages derived from gut microbiota-depleted (white) mice and control (black) mice to phagocytose Streptococcus pneumoniae ex vivo for 30 min. (B) Capacity of whole-blood neutrophils derived from gut microbiota-depleted (white) mice and control (black) mice to phagocytose S. pneumoniae ex vivo for 30 min. (C) Capacity of peritoneal macrophages derived from gut microbiota-depleted (white) mice and control (black) mice to phagocytose $S$. pneumoniae ex vivo for 10 and $30 \mathrm{~min}$. ( $\mathrm{D}$ and $\mathrm{E}$ ) Responsiveness of alveolar macrophages derived from gut microbiota-depleted (white) mice and control (black) mice towards lipoteichoic acid (LTA) and (F and G) lipopolysaccharide (LPS) in terms of interleukin (IL)-6 and tumour necrosis factor (TNF)- $\alpha$ production. (H) TNF- $\alpha$ production of whole blood derived from gut microbiota-depleted (white) mice and control (black) mice upon stimulated with LPS. (I and J) LTA-stimulated peritoneal macrophages derived from gut microbiota-depleted mice (white) and controls (black). (K and L) LPS-stimulated peritoneal macrophages derived from gut microbiota-depleted mice (white) and controls (black). Group size is 8 per group; results are shown as means \pm SEM; n.s. denotes not significant; ${ }^{*} p<0.05,{ }^{* *} p<0.01$ and ${ }^{* * *} p<0.001$.

defences against infections, but also modulates immune responses at systemic sites. ${ }^{7}$ 11-13 21 Clarke et al were among the first to demonstrate that systemic innate immune responses can be enhanced by translocation of microbiota-derived products from the intestine. Bone marrow-derived neutrophils obtained from gut microbiota-depleted mice are less capable of killing S. pneumoniae and Staphylococcus aureus in vitro. ${ }^{7}$ Similarly, intestinal microbiota-primed alveolar macrophages show increased reactive oxygen species-mediated killing of Klebsiella pneumoniae. ${ }^{22}$ In this line, the microbiota has been shown to regulate immune defences against respiratory tract influenza A virus infection. ${ }^{23}{ }^{24}$ Trompette et al ${ }^{11}$ established the existence of a gut-lung axis in allergic airway disease by showing that gut microbiota metabolism of dietary fibres influences the severity of allergic inflammation. Alveolar macrophages, which are resident pulmonary macrophages, are thought to form the first line of defence in the event of pathogen invasion towards the lung. ${ }^{4}{ }^{15}$ We studied the effect of intestinal microbiota depletion on the alveolar macrophage transcriptome. This enabled us to demonstrate that the gut microbiota has a marked influence on metabolic pathways within alveolar macrophages, which correlated with an altered cellular responsiveness-as reflected by a diminished capacity to phagocytose $S$. pneumoniae and a reduced cellular responsiveness towards LTA and LPS. It remains to be established, however, which gut microbiota-derived factors are responsible for this effect. In addition, we did not investigate whether faecal transplantation could reverse the specific transcriptomic changes, impaired immune effector functions of alveolar macrophages and neutrophil influx in our model. Lastly, it remains to be determined whether the observed effects of gut microbiota depletion during infection with $S$. pneumoniae also apply to infections with other important causative agents of pneumonia. Clearly, independent experiments are required to establish this.

Of potential clinical importance is our finding that the gut microbiota protects against organ failure during $S$. pneumoniae-induced 
sepsis. This is illustrated by enhanced liver and hepatic injury in mice in which the gut microbiota was depleted before intranasal infection with $S$. pneumoniae when compared with controls. Interventions to modulate the microbiota composition in the intestine by replacing the microbiota or by treating patients with selected microbial products are promising new treatment strategies for the critically ill. ${ }^{9}$ A very recent report described the first successful use of FMT in the treatment of a critically ill patient with therapy-resistant sepsis. ${ }^{25}$ The finding that Lactobacillus rhamnosus administration is safe in critically ill patients and efficacious for the prevention of ventilator-associated pneumonia further underscores the therapeutic potential of targeting the gut microbiota in pneumonia-derived sepsis. ${ }^{26}$

Caution is needed when extrapolating data from mouse experiments to human disease. Murine models like the one used here make use of a homogenous group of experimental animals with identical genotype, sex and (relatively young) age which are exposed to a well-controlled bacterial challenge, whereas patients form a heterogeneous group in which multiple factors modify disease outcome, including the extent of pathogen exposure, older age, comorbidities, comedications and genetic composition. In addition, it should be noted that FMT in gut microbiota-depleted mice did not reverse all endpoint parameters in our model of bacterial pneumonia. Despite these caveats, the present study now describes a possible role of the gut microbiota in modulating both local and systemic host responses to a clinically relevant model of pneumonia. Our work demonstrates that the intestinal microbiota plays a protective role in the host defence against $S$. pneumonia-induced pneumonia through priming of alveolar macrophages. These results highlight the possibility that broad-spectrum antibiotics and disruption of the intestinal microbiota may diminish innate immune defences to infection. Our data identify a gut-lung axis during infection and establish a mechanism for pulmonary immunomodulation by the intestinal microbiota.

\section{MATERIALS AND METHODS \\ Mice}

Specific pathogen-free $\mathrm{C} 57 \mathrm{Bl} / 6$ mice were purchased from Harlan Laboratories. The known variation in intestinal microbiota composition between inbred C57BL/6 mice derived from one commercial vendor is known to be highly limited. ${ }^{27}$ Offspring and diet are major determinants of gut microbiota composition. $^{28} 29$ Experimental groups were age and sex matched, and housed in the Animal Research Institute Amsterdam (ARIA) facility of the Academic Medical Center facility under standard care. All experiments were conducted with mice between 10 and 12 weeks of age.

\section{Experimental study design}

Pneumonia was induced by intranasal inoculation with $1 \times 10^{6}$ CFU S. pneumoniae D39 as previously described. ${ }^{30-32}$ For survival experiments, mice were checked every $6 \mathrm{~h}$ until death occurred. Sample harvesting, determination of bacterial growth and analysis (including pathology and immunohistochemistry) are described in the online supplementary file. In order to deplete the gut microbiota, mice were treated with broadspectrum antibiotics (ampicillin $1 \mathrm{~g} / \mathrm{L}$, Sigma; neomycin sulfate $1 \mathrm{~g} / \mathrm{L}$, Sigma; metronidazole $1 \mathrm{~g} / \mathrm{L}$, Sanofi-Aventis and vancomycin $0.5 \mathrm{~g} / \mathrm{L}$, Sandoz) in drinking water for 3 weeks as described. ${ }^{7}$ Two days after cessation of antibiotic drinking water, mice were inoculated with $S$. pneumoniae. RNA preparation from alveolar macrophages and microarray profiling are described in the online supplementary file.

\section{Faecal transplantation}

Faecal pellets from untreated mice were resuspended in PBS (1 faecal pellet/ $1 \mathrm{~mL}$ of PBS). For each experiment, several faecal pellets from different untreated mice were resuspended together in PBS. A total of $200 \mu \mathrm{L}$ of the resuspended pool faecal material was given by oral gavage to gut microbiota-depleted mice over 4 consecutive days after antibiotic treatment was stopped; control mice were given water alone during this period. ${ }^{33}$

\section{Microbiota analyses}

Fresh stool pellets were obtained before mice were euthanised. The samples were immediately frozen and stored at $-80^{\circ} \mathrm{C}$. DNA isolation was performed using a modified repeated beating method. $^{3435} 16 \mathrm{~S}$ rRNA gene amplification, in vitro transcription and labelling and hybridisation were carried out as described previously. ${ }^{17}$ Data analyses were performed using the microbiome $\mathrm{R}$-script package as described in the online supplementary file.

\section{Ex vivo experiments}

Murine alveolar macrophages and neutrophils were isolated, washed and incubated as described. ${ }^{36} 37$ Details on the phagocytosis and cell stimulation experiments are provided in the online supplementary file.

\section{Ethics statement}

The Institutional Animal Care and Use Committee of the Academic Medical Center, University of Amsterdam, reviewed and approved all experiments (identification number: DIX100121AE). The animal care and use protocol adhered to European Directive of 22 September 2010 (Directive 2010/63/ $\mathrm{EU}$ ) in addition to the Directive of 6 May 2009 (Directive 2009/41/EC).

\section{Statistical analysis}

Comparisons between groups were first performed using Kruskal-Wallis one-way analysis of variance (ANOVA) test; in case of significant differences, differences between groups were tested using the Mann-Whitney U test (GraphPad Prism 5). We used the Kaplan-Meier log-rank test to compare survival between groups. Statistically significant differences are indicated as follows: $* \mathrm{p}<0.05 ; * * \mathrm{p}<0.01 ; * * \mathrm{p}<0.001$. Linear model ANOVA was employed to define alveolar macrophage transcripts influenced by broad-spectrum antibiotic treatment. Unless otherwise stated, a false discovery rate-corrected $\mathrm{p}$ value (q-value) was used to define genome-wide significance.

\section{Author affiliations \\ ${ }^{1}$ Center for Experimental and Molecular Medicine, Academic Medical Center University of Amsterdam, Amsterdam, The Netherlands \\ ${ }^{2}$ Department of Medicine, Division of Infectious Diseases, Academic Medical Center, University of Amsterdam, Amsterdam, The Netherlands \\ ${ }^{3}$ Department of Clinical Chemistry, Hematology and Immunology, Diakonessenhuis Utrecht, The Netherlands \\ ${ }^{4}$ Department of Pathology, Academic Medical Center, University of Amsterdam, Amsterdam, The Netherlands \\ ${ }^{5}$ Laboratory of Microbiology, Wageningen University, Wageningen, The Netherlands ${ }^{6}$ Department of Bacteriology \& Immunology, Helsinki University, Helsinki, Finland}

Acknowledgements We thank Marieke ten Brink and Joost Daalhuisen for technical support and Professor Gijs van den Brink (Department of Gastroenterology, Academic Medical Center, University of Amsterdam) for fruitful discussions.

Contributors TJS designed and did experiments, analysed data and wrote the paper; WJW conceived ideas, wrote the paper and oversaw the research programme; $J M L, B P S, J J T H R, J D d B, A J H, A d V$ and CB. designed and did experiments and analysed data; RdB and FdSeM did experiments; TJS and BPS performed bioinformatics analysis; WMdV and TvdP provided advice and oversaw a portion of the work. 
Funding This work was supported by the Netherlands Organization for Scientific Research (NWO) and The Netherlands Organization for Health Research development (ZonMw).

Competing interests None declared.

Provenance and peer review Not commissioned; externally peer reviewed.

Open Access This is an Open Access article distributed in accordance with the Creative Commons Attribution Non Commercial (CC BY-NC 4.0) license, which permits others to distribute, remix, adapt, build upon this work non-commercially, and license their derivative works on different terms, provided the original work is properly cited and the use is non-commercial. See: http://creativecommons.org/ licenses/by-nc/4.0/

\section{REFERENCES}

1 Wunderink RG, Waterer GW. Clinical practice. Community-acquired pneumonia. N Engl J Med 2014;370:543-51.

2 Nair H, Simoes EA, Rudan I, et al. Global and regional burden of hospital admissions for severe acute lower respiratory infections in young children in 2010: a systematic analysis. Lancet 2013;381:1380-90.

3 van der Poll T, Opal SM. Pathogenesis, treatment, and prevention of pneumococcal pneumonia. Lancet 2009;374:1543-56.

4 Dockrell DH, Whyte MK, Mitchell TJ. Pneumococcal pneumonia: mechanisms of infection and resolution. Chest 2012;142:482-91.

5 Clark JA, Coopersmith CM. Intestinal crosstalk: a new paradigm for understanding the gut as the "motor" of critical illness. Shock 2007;28:384-93.

6 Clemente JC, Ursell LK, Parfrey LW, et al. The impact of the gut microbiota on human health: an integrative view. Cell 2012:148:1258-70.

7 Clarke TB, Davis KM, Lysenko ES, et al. Recognition of peptidoglycan from the microbiota by Nod1 enhances systemic innate immunity. Nat Med 2010;16:228-31.

8 Noverr MC, Huffnagle GB. Does the microbiota regulate immune responses outside the gut? Trends Microbiol 2004;12:562-8.

9 Schuijt TJ, van der Poll T, de Vos WM, et al. The intestinal microbiota and host immune interactions in the critically ill. Trends Microbiol 2013;21:221-9.

10 Backhed F, Fraser $\mathrm{CM}$, Ringel $\mathrm{Y}$, et al. Defining a healthy human gut microbiome: current concepts, future directions, and clinical applications. Cell Host Microbe 2012:12:611-22.

11 Trompette A, Gollwitzer ES, Yadava K, et al. Gut microbiota metabolism of dietary fiber influences allergic airway disease and hematopoiesis. Nat Med 2014;20:159-66

12 Deshmukh HS, Liu Y, Menkiti OR, et al. The microbiota regulates neutrophil homeostasis and host resistance to Escherichia coli $\mathrm{K} 1$ sepsis in neonatal mice. Nat Med 2014;20:524-30.

13 Caballero S, Pamer EG. Microbiota-mediated inflammation and antimicrobial defense in the intestine. Annu Rev Immunol 2015;33:227-56.

14 Dethlefsen L, Relman DA. Incomplete recovery and individualized responses of the human distal gut microbiota to repeated antibiotic perturbation. Proc Natl Acad Sci USA 2011;108(Suppl 1):4554-61.

15 Strieter RM, Belperio JA, Keane MP. Cytokines in innate host defense in the lung. J Clin Invest 2002;109:699-705.

16 Arumugam M, Raes J, Pelletier E, et al. Enterotypes of the human gut microbiome. Nature 2011:473:174-80

17 Rajilic-Stojanovic M, Heilig HG, Molenaar D, et al. Development and application of the human intestinal tract chip, a phylogenetic microarray: analysis of universally conserved phylotypes in the abundant microbiota of young and elderly adults. Environ Microbiol 2009;11:1736-51.
18 Serezani $\mathrm{CH}$, Aronoff DM, Sitrin RG, et al. FcgammaRI ligation leads to a complex with BLT1 in lipid rafts that enhances rat lung macrophage antimicrobial functions. Blood 2009;114:3316-24

19 Kannan S, Audet A, Huang $\mathrm{H}$, et al. Cholesterol-rich membrane rafts and Lyn are involved in phagocytosis during Pseudomonas aeruginosa infection. I Immunol 2008;180:2396-408.

20 Angus DC, van der Poll T. Severe sepsis and septic shock. N Eng/ J Med 2013:369:2063.

21 Blaser M, Bork P, Fraser C, et al. The microbiome explored: recent insights and future challenges. Nat Rev Microbiol 2013;11:213-17.

22 Clarke TB. Early innate immunity to bacterial infection in the lung is regulated systemically by the commensal microbiota via nod-like receptor ligands. Infect Immun 2014:82:4596-606.

23 Ichinohe T, Pang IK, Kumamoto $Y$, et al. Microbiota regulates immune defense against respiratory tract influenza A virus infection. Proc Natl Acad Sci USA 2011;108:5354-9.

24 Wang J, Li F, Sun R, et al. Bacterial colonization dampens influenza-mediated acute lung injury via induction of M2 alveolar macrophages. Nat Commun 2013;4:2106.

25 Li Q, Wang C, Tang C, et al. Therapeutic modulation and reestablishment of the intestinal microbiota with fecal microbiota transplantation resolves sepsis and diarrhea in a patient. Amer J of Gastroenter 2014;109:1832-4.

26 Morrow LE, Kollef MH, Casale TB. Probiotic prophylaxis of ventilator-associated pneumonia: a blinded, randomized, controlled trial. Am J Respir Crit Care Med 2010;182:1058-64.

27 Ericsson AC, Davis JW, Spollen W, et al. Effects of vendor and genetic background on the composition of the fecal microbiota of inbred mice. PLOS ONE 2015;10: e0116704.

28 Carmody RN, Gerber GK, Luevano JM Jr, et al. Diet dominates host genotype in shaping the murine gut microbiota. Cell Host Microbe 2015;17:72-84.

29 Tilg H, Moschen AR. Microbiota and diabetes: an evolving relationship. Gut 2014;63:1513-21.

30 Rijneveld AW, Florquin $\mathrm{S}$, Bresser $\mathrm{P}$, et al. Plasminogen activator inhibitor type-1 deficiency does not influence the outcome of murine pneumococcal pneumonia. Blood 2003;102:934-9.

31 van der Windt GJ, Blok DC, Hoogerwerf JJ, et al. Interleukin 1 receptor-associated kinase $\mathrm{m}$ impairs host defense during pneumococcal pneumonia. J Infect Dis 2012:205:1849-57.

32 Duitman J, Schouten M, Groot AP, et al. CCAAT/enhancer-binding protein delta facilitates bacterial dissemination during pneumococcal pneumonia in a platelet-activating factor receptor-dependent manner. Proc Natl Acad Sci USA 2012;109:9113-18

33 Ubeda C, Bucci V, Caballero S, et al. Intestinal microbiota containing Barnesiella species cures vancomycin-resistant Enterococcus faecium colonization. Infect Immun 2013:81:965-73.

34 Ubeda C, Taur Y, Jenq RR, et al. Vancomycin-resistant Enterococcus domination of intestinal microbiota is enabled by antibiotic treatment in mice and precedes bloodstream invasion in humans. J Clin Invest 2010;120:4332-41.

35 Salonen A, Nikkila J, Jalanka-Tuovinen J, et al. Comparative analysis of fecal DNA extraction methods with phylogenetic microarray: effective recovery of bacterial and archaeal DNA using mechanical cell lysis. J Microbiol Methods 2010;81:127-34.

36 Wiersinga WJ, Kager LM, Hovius JW, et al. Urokinase receptor is necessary for bacterial defense against pneumonia-derived septic melioidosis by facilitating phagocytosis. J Immunol 2010;184:3079-86.

37 Kager LM, Weehuizen TA, Wiersinga WJ, et al. Endogenous alpha2-antiplasmin is protective during severe gram-negative sepsis (melioidosis). Am J Respir Crit Care Med 2013;188:967-75. 